\title{
More options for prostate cancer
}

With the advent of new therapies in the treatment of prostate cancer, the therapeutic landscape is becoming increasingly cluttered. This increased choice of therapies for patients with metastatic castration-resistant prostate cancer (mCRPC) has been added to by a new phase III clinical trial showing that the previous standard of care-high-dose 3-weekly docetaxel in combination with prednisone-can be replaced with a lowerdose 2-weekly docetaxel combination without detriment to patients.

The rationale of this phase III, randomized trial was the possibility of an improved safety profile for the 2-weekly regimen in the first-line setting, as explained by lead author Pirkko-Liisa Kellokumpu-Lehtinen: "if we can avoid severe neutropenia, which is related to neutropenic infections (and hospitalizations due to infections), the patients might be able to continue treatment for a longer time."

As the investigators had predicted, fewer grade 3 and 4 adverse effects were noted in the 2-weekly group. Furthermore, the primary end point of significantly improved time to treatment failure was also met in the 2-weekly group (5.6 months versus 4.9 months). In an accompanying commentary, David Nanus and Scott Tagawa pointed out that "this regimen is a reasonable alternative to the standard regimen." However, they also noted that although the limitations of the study would usually require confirmation, they do not believe that it would be the best use of limited resources.

Kellokumpu-Lehtinen plans to use "serum and tissue samples taken during the trial to test for possible predictive and prognostic factors," in addition to testing the regimen in combination with newer chemotherapy agents.

\section{Rebecca Kirk}

Original article Kellokumpu-Lehtinen, P.-L. et al. 2-weekly versus 3-weekly docetaxel to treat castration-resistant advanced prostate cancer: a randomised, phase 3 trial. Lancet Oncol. doi:10.1016/S1470-2045(12)70537-5 